A SIMPLE, INEXPENSIVE DIPOLE SPECTRONETER FOR ISABBLLE *

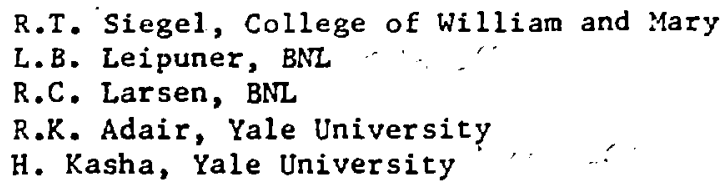

The nagnets from obsolete large cyclotrons have been found to be extremely useful as spectrometer magnets at high energy physics laboracories. The Chicago cyclotron magnet is the basis of a large spectrometer at Fermilab, where very inceresting measurements of muon production and interactions have been conducted. Fermilab is now considering the acquisition of the Nevis cyclotron magnet as the basis of another di-muon and hadron spectrometer. The magnet from the Carnegie Institute of Technology cyclotron has been transported to CERN where it is to be used as a spectrometer magnet at the ISR. We propose to acquire the Space Radiation Effects Laboratory (SREL) 5-m cyclotron magnet for use as the basis of a large, simple dipole spectrometer at an ISABELIE colliding-beam intersection.

The magnet (Fig. 1) consists of about 2700 tons of steel and two 55-ton aluminum coils. The steel is in 54 major pieces each weighing about 50 tons. The magnet was assembled, and can be disassembled, by using a crane rated at 50 tons (which could be used, carefully, at an ove eload to lift the coils). The original assembly was straightforward, and, since we have all the relevant engineering information, the proposed disassembly and future assembly at ISABELLE should not be especially difficult. Transportation of the material from Newport News to Brookhaven, while hardly a trivial

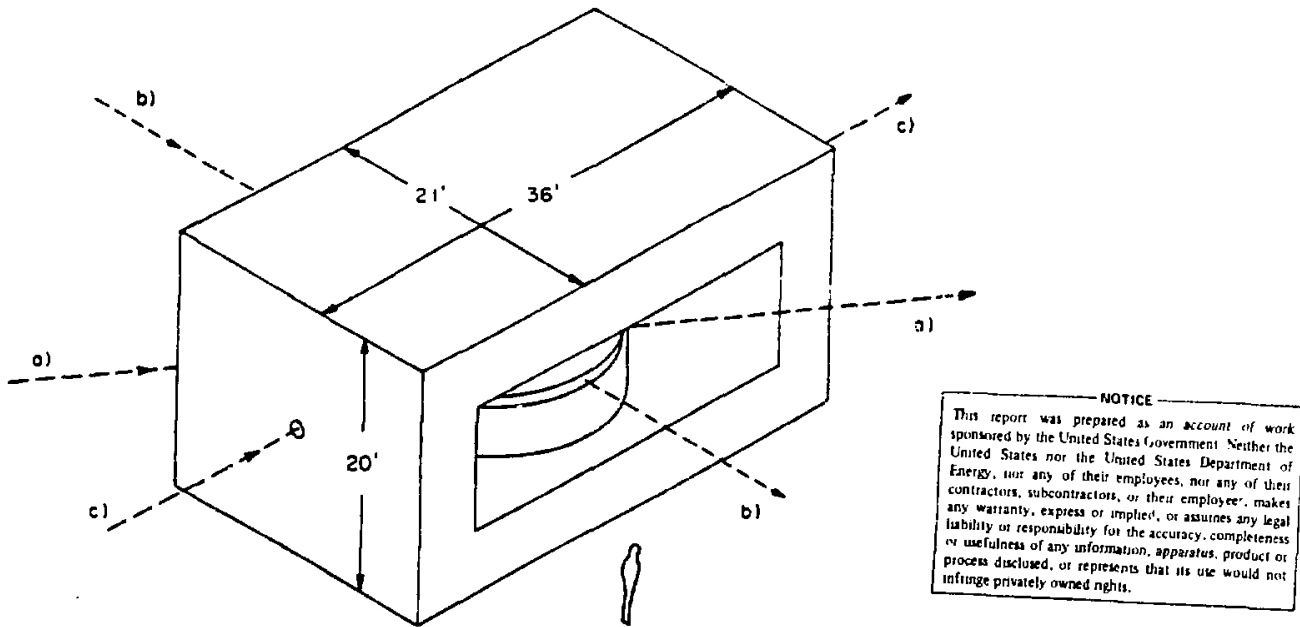

Fig. 1. Schematic view of cyclotron magnet. Lines a), b), and c) represent different possible proton beam trajectories.

*Supported in part by the United States Department of Energy. 
enterprise, should present no unusual difficulties. With the rather large amount of aluminum in the coils, the magnet operates at full load using only $1.2 \mathrm{MW}$ of power. The power supply is available, and we propose to bring it to BNL along with the heat exchanger system used for the contained cooling water. The 50-ton crane is also availabie, as well as 1500 tons of steel shielding in the form of 12012.5 -ton blocks. Careful estimates indicate that the disassembly costs will be about $\$ 125,000$, and the assembly costs should not be much different. Thus the cost of removing the magnet from SREL in Virginia and bringing it to BNL should be $\ll 300,000$. The cost of setting it up at the ISABELLE intersection should not be $>\$ 200,000$, bringing the total to about $\$ 500,000$. The gap of the magnet, as designed, is $18^{\prime \prime}$ without the shaped pole tips, which we would not plan to use. This gap can be expanded to 42 " by removing one pole plate from the top and the bottom of the magnet. Intermediate gaps can be achieved easily at the cost of the machining of extra pole plates. At full power, the field will be about $19.5 \mathrm{kG}$ at the smaller gap and about $14.5 \mathrm{kG}$ for the 42" gap. Since we plan to use the magnet in a manrer such that the intersection collisions take place near the center of the field, we must shield the beam from that field. We propose to run the beam through a superconducting tube about 40' long which will exclude the field from the beam trajectory region. This field exclusion method has been used successfully at SLAC.

We wish to propose the magnet as the basic instrument of a flexible spectrometer facility, and we would then like to consider that the magnet might be used in different configurations with respect to the beam direction. Measurements of muons are best made in a manner such that the muons pass through the back legs of the magnet, which are then excellent spectrometer magnets themselves. For applications involving the spectrometry of hadrons, the back legs can prove to be an obstacle. Thus, to measure muons and hadrons with maximum flexibility, it is desirable to have the possibility of arranging the magnet at different angles with respect to the proton beam direction. Figure 1 shows three possible directions of the beam with respect to the magnet orientarion. We would then hope to place the magnet in such a way that it can be rotated without excessive effort. We suggest mounting the magnet on oil pads, for example. Since the current supplied to the magnet is only about $2000 \mathrm{~A}$ at $600 \mathrm{~V}$, and the magnet cooling requires only about $200 \mathrm{gpm}$ of water, the power and cooling water connections can be changed easily.

It is desirable to consider specific experiments that might be conducted at any proposed facility. Since, at the rate defined by the present funding schedule, ISABELLE will not operate until 1985, it is not practical to attempt to design a detailed experiment. We do not really know what questions will be important by then, nor do we have a good idea of the technology that will be available in 7 years. Nevertheless, it is useful to consider the general character of some designs of experiments that might provide answers to current questions by techniques already available in order to 

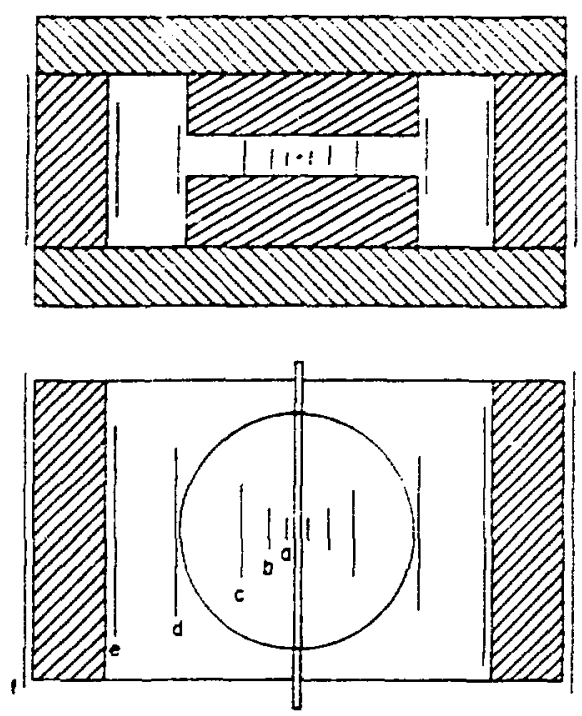

Fig. 2. Suggested detector disposition for $W$ and $Z$ detection experiments. Typical detector dimensions: (a) $24 \times 12^{\prime \prime}$ PWC; (b) 36 $\times 18^{\prime \prime}$ PWC; (c) $30 \times 72^{\prime \prime}$ PWC; (d) $12 \times 6^{\prime}$ PWC; (e) $15 \times 7^{\prime}$ drift chambers; (f) $24 \times 12^{\prime}$ drift chambers.

demonstrate the flexibility and power (and limitations) of the facility. We present a conceptual design of experiments designed to measure the production of the $W$ anc the $Z$, and the spectrometer part of experiments designed to measure the quark-quark differential scattering cross sections through the measurement of jet-jet energy and angular distributions.

Figure 2 shows the configuration of magnet and detectors which rould be used in measurements of cross sections ror production of $W$ and $Z$ bosons at large angles. The large-angle cross sections are attractive because thexe is less likelihood of background problems due to muon pairs produced through conventional mechanisms. Here we accept production over an interval from $55^{\circ}$ to $125^{\circ}$ with an azimuthal acceptance of about $24 \%$ of the $2 \pi$ available angle. With use of completely conventional techniques based on proportional wire chamber detectors with conservative $2-\operatorname{mm}$ wire spacing, the muon momentum resolution will be about $100 \% \times \mathrm{p}$, with $\mathrm{p}$ measured in $\mathrm{TeV}$. The cost of the instrumentation, excluding the magnet but including all electronics and an on-line computer, will be about $\$ 1,000,000$. Assuming a canonical luminosity of $10^{33}$, the counting rate for muons from $W$-decay might be expected to be of the order of $-v 0$ per hour. The rate of muon pairs from $z$-decay is less easily estimated but should be smaller by not less than an order of magnitude, or of the order of 10 per hour.

We are now beginning to gain some appreciation for the character of the jets resulting from the production of high energy 


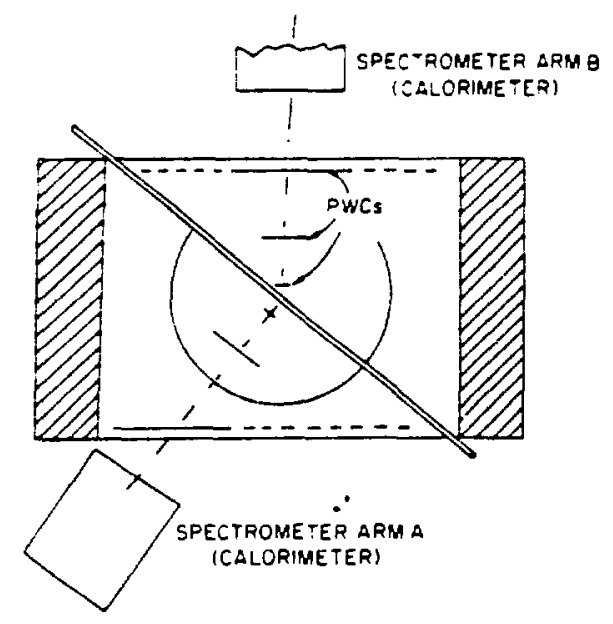

Fig. 3. Magnet use as double-arm spectrometer for jet study.

partons--and, perhaps, high energy gluons. The spectrometer, augmented by appropriate calorimetry (such as that used by Selove, Irwin, and their collaborators at Fermilab), would appear to be a most useful tool for the measurement of jet-jet energy and angular correlations and then, perhaps, quark-quark scattering angular distributions and gluon-gluon scattering angular distributions. (See Fig. 3.) Here we would limit ourselves to jets with a considerable total energy produced at relatively large angles $\left(e . g_{0},>5^{\circ}\right.$ ) with respect to the proton direction, and we might find it best to run the magnet below the maximum fields. The neutral particles and the high energy charged particles would be captured in the calorimeter; the lower energy charged particles would be deflected by the field but their energies would be accurately measured through the measurement of their curvature by the PWCs. With the total momentun of each jet known, one can deduce the momenta of the interacting particles, partons or gluons, with no important error.

In general, the spectrometer would be basic to the single-arm spectrometer extensions requix'ed for measurements of inclusive hadron spectra as well as multiarm spectrometers designed to record more complicated correlations. We would hope that the facility would form the base for an area in which modest programs could be developed. 


\title{
A SIMPLE, INEXPENSIVE DIPOLE SPECTROMETER FOR ISABELLE *
}

\author{
R.T. Siegel, College of William and Mary \\ L.B. Leipuner, BNL \\ R.C. Larsen, BNL \\ R.K. Adair, Yale University \\ H. Kasha, Yale University
}

The magnets from obsolete large cyclotrons have been found to be extremely useful as spectrometer magnets at high energy physics laboratories. The Chicago cyclotron magnet is the basis of a large spectrometer at Fermilab, where very interesting measurements of muon production and interactions have been conducted. Fermilab is now considering the acquisition of the Nevis cyclotron magnet as the basis of another di-muon and hadron spectrometer. The magnet from the Carnegie Institute of Technology cyclotron has been transported to CERN where it is to be used as a spectrometer magnet at the ISR. We propose to acquire the Space Radiation Effects Laboratory (SREL) 5-m cyclotron magnet for use as the basis of a large, simple dipole spectrometer at an ISABELLE colliding-beam intersection.

The magnet (Fig. 1) consists of about 2700 tons of steel and two 55-ton aluminum coils. The steel is in 54 major pieses each weighing about 50 tons. The magnet was assembled, and can be disassembled, by using a crane rated at 50 tons (which could be used, carefully, at an overload to lift the coils). The original assembly was straightforward, and, since we have all the relevant engineering information, the proposed disassembly and furure assembly at ISABELLE should not be especially difficult. Transportation of the material from Newport News to Brookhaven, while hardly a trivial

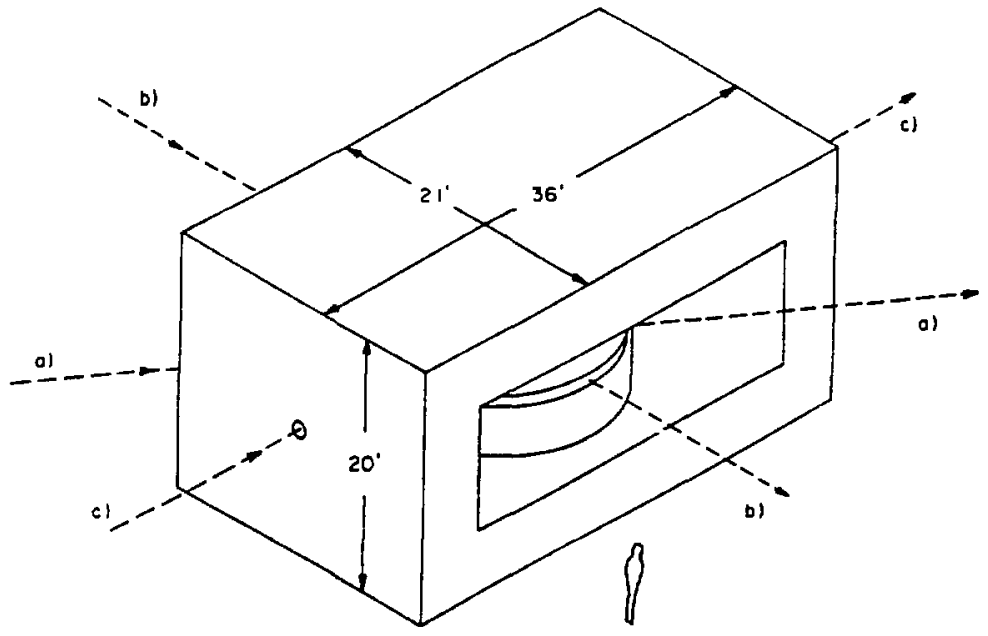

Fig. 1. Schematic view of cyclotron magnet. Lines a), b), and c) represent different possible proton beam trajectories.

tSupported in part by the United States Department of Energy.. 
enterprise, should present no unusual difficulties. With the racher large amount of aluminum in the coils, the magnet operates at full load using only $1.2 \mathrm{MW}$ of power. The power supply is available, and we propose to bring it to BNL along with the heat exchanger system used for the contained cooling water. The 50-ton crane is also available, as well as 1500 tons of steel shielding in the form of 12012.5 -ton blocks. Careful estimates indicate that the disassembly costs will be about $\$ 125,000$, and the assembly costs should not be much different. Thus the cost of removing the magnet from SREL in Virginia and bringing it to BNL should be $<3300,000$. The cost of setting it up at the ISABELLE intersection should not be $>\$ 200,000$, bringing the total to about $\$ 500,000$.

The gap of the magnet, as designed, is 18" without the shaped pole tips, which we would not plan to use. This gap can be expanded to 42 " by removing one pole plate from the top and the bottom of the the $t$. Intermediate gaps can be achieved easily at the cost of the machining of extra pole plates. At full power, the field will be about $19.5 \mathrm{kG}$ at the smaller gap and about $14.5 \mathrm{kG}$ for the 42" gap. Since we plan to use the magnet in a manner such that the intersection collisions take place near the center of the field, we must shield the beam from that field. We propose to run the beam through a superconducting tube about $40^{\prime}$ long which will exclude the field from the beam trajectory region. This field exclusion method has been used successfully at SLAC.

We wish to propose the magnet as the basic instrument of a flexible spectrometer facility, and we would then like to consider that the magnet might be used in different configurations with respect to the beam direction. Measurements of muons ar best made in a manner such that the muons pass through the back legs of the magnet, which are then excellent spectrometer magnets themselves. For applications involving the spectrometry of hadrons, the back legs can prove to be an obstacle. Thus, to measure muons and hadrons with maximum flexibility, it is desirable to have the possibility of arranging the magnet at different angles with respect to the proton beam direction. Figure 1 shows three possible directions of the beam with respect to the magnet orientation. We would then hope to place the magnet in such a way that it can be rotated without excessive effort. We suggest mounting the magnet on oil pads, for example. Since the current supplied to the magnet is only about $2000 \mathrm{~A}$ at $600 \mathrm{~V}$, and the magnet cooling requires only about $200 \mathrm{gpm}$ of water, the power and cooling water connections can be changed easily.

It 1 is desirable to consider specific experiments that might be conducted at any proposed facility. Since, at the rate defined by the present funding schedule, ISABELLE will not operate until 1985, it is not practical to attempt to design a detailed experiment. We do not really know what questions will be important by then, nor do we have a good idea of the technology that will be available in 7 years. Nevertheless, it is useful to consider the general character of some designs of experiments that might provide answers to current questions by techniques already available in order to 

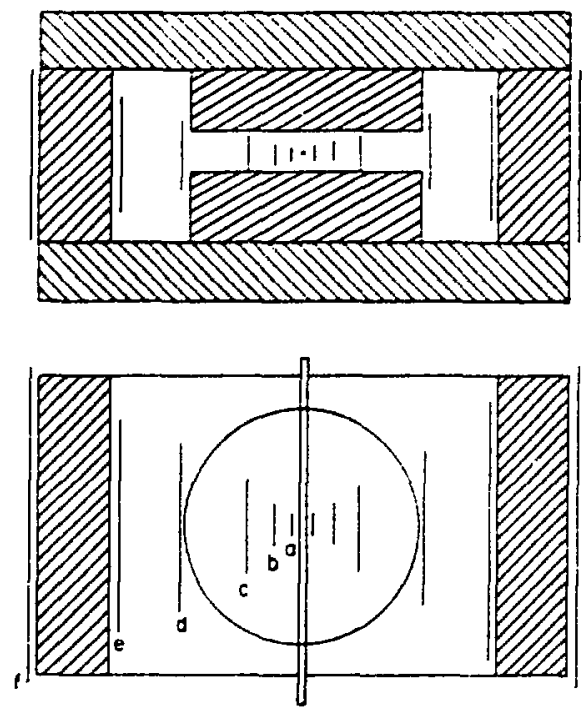

Fig. 2. Suggested detector disposition for $W$ and $Z$ detection experiments. Typical detector dimensions: (a) $24 \times 12^{\prime \prime}$ PWC; (b) 36 $\times 18^{\prime \prime}$ PWC; (c) $30 \times 72^{\prime \prime}$ PWC; (d) $12 \times 6^{\prime}$ PWC; (e) $15 \times 7^{\prime}$ drift chambers; (f) $24 \times 12^{\prime}$ drift chambers.

demonstrate the flexibility and power (and limitations) of the facilicy. We present a conceptual design of experiments designed to measure the production of the $W$ and the $Z$, and the spectrometer part of experiments designed to measure the quark-quark differential scattering cross sections through the measurement of jet-jet energy and angular distributions.

Figure 2 shows the configuration of magnet and detectors which could be used in measurements of cross sections for production of $W$ and $Z$ bosons at large angles. The large-angle cross sections are attractive because there is less likelihood of background problems due to muon pairs produced through conventional mechanisms. Here we accept production over an interval from $55^{\circ}$ to $125^{\circ}$ with an azimuthal acceptance of about $24 \%$ of the $2 \pi$ available angle. With use of completely conventional techniques based on proportional wire chamber detectors with conservative 2-mm wire spacing, the muon momentum resolution will be about $100 \% \times \mathrm{p}$, with $p$ measured in $\mathrm{TeV}$. The cost of the instrumentation, excluding the magnet but including all electronics and an oil-line computer, will be about $\$ 1,000,000$. Assuming a canonical luminosity of $10^{33}$, the counting rates for muons from $W$-decay might be expected to be of the order of 100 per hour. The rate of muon pairs from $z$-decay is less easily estimated but should be smaller by not less than an order of magnitude, or of the order of 10 per hour.

We are now beginning to gain some appreciation for the character of the jets resulting from the production of high energy 